\title{
Identification of Stem Leydig Cells Derived from Pig Testicular Interstitium
}

\author{
Shuai Yu, Pengfei Zhang, Wuzi Dong, Wenxian Zeng, and Chuanying Pan \\ College of Animal Science and Technology, Northwest A\&F University, Yangling, Shaanxi 712100, China \\ Correspondence should be addressed to Chuanying Pan; chuanyingpan@126.com
}

Received 26 August 2016; Accepted 19 December 2016; Published 24 January 2017

Academic Editor: James Adjaye

Copyright ( 2017 Shuai Yu et al. This is an open access article distributed under the Creative Commons Attribution License, which permits unrestricted use, distribution, and reproduction in any medium, provided the original work is properly cited.

\begin{abstract}
Stem Leydig cells (SLCs), located in the testicular interstitial compartment in the mammalian testes, are capable of differentiating to testosterone-synthesizing Leydig cells (LCs), thus providing a new strategy for treating testosterone deficiency. However, no previous reports have identified and cultured SLCs derived from the pig. The aim of the current study was to isolate, identify, and culture SLCs from pigs. Haematoxylin and eosin staining and immunochemical analysis showed that SLCs were present and that PDGFR $\alpha$ was mainly expressed in the pig testicular interstitium, indicating that PDGFR $\alpha$ was a marker for SLCs in the neonatal pig. In addition, reverse transcription-PCR results showed that SLC markers were expressed in primary isolated LCs, indicating that they were putative SLCs. The putative SLCs were subsequently cultured with a testicular fluid of piglets (pTF) medium. Clones formed after 7 days and the cells expressed PDGFR $\alpha$. However, no clones grew in the absence of pTF, but the cells expressed CYP17A1, indicating that pTF could sustain the features of porcine SLCs. To summarize, we isolated porcine SLCs and identified their basic characteristics. Taken together, these results may help lay the foundation for research in the clinical application of porcine SLCs.
\end{abstract}

\section{Introduction}

Testosterone not only maintains the spermatogenesis process, but also protects the function of androgen-dependent tissues $[1,2]$. As the primary source of synthesizing and secreting testosterone in mammalian testes, adult Leydig cells (ALCs) play essential roles in maintaining vital movement [3]. Recently, it has been demonstrated that ALCs arise from stem Leydig cells (SLCs) [4]. SLCs, which are located in the interstitial compartment close to the seminiferous tubules in mammalian testes, are one of the most important somatic stem cells types $[5,6]$.

SLCs were firstly identified and enriched from neonatal rat testes by Ge et al. (2006), and further studies demonstrated that putative mouse and human SLCs had the capacity to differentiate into testosterone-producing cells $[4,7,8]$. According to these previous studies, some characteristics of SLCs were identified $[4,9,10]$. First, the number of mammalian SLCs was fairly small; for example, an average of only 8,500 putative SLCs were obtained from one postnatal 7day-old rat testes [4]. Second, the SLCs residing on the outer surface of the seminiferous tubules in rat testes were spindleshaped in situ $[4,10]$. In addition, putative SLCs expressed LIF receptor (LIFR), platelet-derived growth factor receptor $\alpha$ (PDGFR $\alpha$ ), Nestin, Thy-1, and some stem cell markers; however, they were $3 \beta$-HSD- and luteinizing hormone receptor(LHR-) negative $[4,8,10,11]$. Unfortunately, no SLCs studies had been carried out in other mammalian animals except in rats, mice, and humans.

With increasing age, the number of functional LCs decreased, and the ability for testosterone production, cAMP production, and the activities of steroidogenic enzymes is reduced $[12,13]$. Thus, male infertility diseases may occur in older males as a result of LCs dysfunction or testosterone disorder $[14,15]$. Currently, androgen-replacement was the most efficient therapy for rescuing testosterone deficiency; however, it required successive treatments and carries inherent risks [16]. SLCs had the ability to self-renew and differentiate into LCs, therefore, providing a new strategy for treating these diseases by SLCs transplantation [17].

The pig had played a crucial role as a mammalian model in human disease studies $[18,19]$. The pig testis had 
been suggested as "the most versatile steroid producing organ known" and provided important material to research the physiology and genetics of human steroidogenesis [20]. However, porcine SLCs had yet to be isolated and enriched. Additionally, species distinctions complicated the studies of porcine SLCs, since completely mapping of the markers and culture systems of rat and mouse SLCs to porcine SLCs had not been achieved. Owing to the importance of porcine SLCs in clinical applications, the objective of this study was to isolate, identify, and culture SLCs from neonatal pig testes.

\section{Materials and Methods}

2.1. Collection of Porcine Testes. The study was approved by the Animal Care and Use Committee of Northwest A\&F University in accordance with the Guide for the Care and Use of Laboratory Animals of the National Institutes of Health, China. Fresh testes samples of 7-day-old male pigs from Besun agricultural industry group Co., Ltd. (Yangling, Shaanxi, China) were transported to the laboratory in Dulbecco's phosphate-buffered saline (DPBS) supplemented with $2 \%$ Penicillin-Streptomycin (P/S) solution (Invitrogen, Carlsbad, CA, USA) within $1 \mathrm{~h}$. Testes samples of 2-monthold male pigs were collected from a pig breeding farm in Yangling, Shaanxi Province, China.

2.2. Isolation of Porcine SLCs. An enzymatic digestion method was used for obtaining porcine SLCs. Testes were first washed and minced after the epididymis and tunica albuginea were removed. Then the testicular fragments were suspended in $0.75 \mathrm{mg} / \mathrm{mL}$ collagenase type IV (Invitrogen) containing $5 \%(\mathrm{v} / \mathrm{v})$ fetal bovine serum (FBS, Gibco, UK) plus DNase I $(100 \mu \mathrm{g} / \mathrm{mL}$; Bio Basic, Markham, Canada) and incubated, with constant shaking at $34^{\circ} \mathrm{C}$ for $90 \mathrm{~min}$ [21]. The 160 and $59 \mu \mathrm{m}$ monofilament nylon meshes (Solarbio, Beijing, China) were then used to filter the cell suspension [21]. The isolated cells were treated with $1 \mathrm{mg} / \mathrm{mL}$ hyaluronidase (Invitrogen) and centrifugation at $500 \mathrm{~g}$ for $5 \mathrm{~min}$ at $20^{\circ} \mathrm{C}$. After $5 \mathrm{~min}$ stilling, the upper side of the suspensions was cultured in media for another $15 \mathrm{~min}$ stilling. The cells on the upper side of the suspensions were then collected. Finally, the isolated LCs were cultured in Dulbecco's modified eagle medium: nutrient mixture F-12 (DMEM/F12, Invitrogen) medium.

2.3. Preparation of Testicular Fluid of Piglets ( $p T F)$. The pTF and primary LCs were derived from the same source. The testes of 7-day-old pigs were cut into fragments as small as possible and pTF was extracted by tissue homogenization at $20^{\circ} \mathrm{C}$ [22]. Finally, the pTF was filtered through a $0.22 \mu \mathrm{m}$ strainer to degerm.

2.4. Culture of Porcine Isolated LCs. The isolated LCs precipitates were resuspended in two media: one basic medium and the other pTF medium (basic medium plus 30\% (v/v) pTF) [22]. The basic medium consisted of DMEM/F12, 10\% (v/v) FBS, $1 \%(\mathrm{v} / \mathrm{v}) \mathrm{P} / \mathrm{S}$, and $1 \%(\mathrm{v} / \mathrm{v})$ vitamins. The LCs were then incubated in an atmosphere of $95 \%$ air- $5 \% \mathrm{CO}_{2}$ at $34^{\circ} \mathrm{C}$ and cultured for at least 2 weeks. The culture media were changed daily.

2.5. Ethane Dimethanesulphonate (EDS) Treatment. The EDS was provided by Professor Yuanqiang Zhang (Department of Human Anatomy, Histology and Embryology, The Fourth Military Medical University, China). According to the previous methods, EDS was dissolved in dimethyl sulfoxide (DMSO)/sterile water (1:3, v/v) [23-25]. Afterwards, the primary isolated SLCs were seeded into a 6-well plate and $0,0.5$, 0.75 , and $1.0 \mathrm{mg} / \mathrm{mL}$ EDS (final concentration) were added to the culture solution, respectively $[24,25]$. Quantitative real time-PCR (qRT-PCR) and immunofluorescent analyses were carried out $24 \mathrm{~h}$ after EDS treatment.

2.6. Haematoxylin and Eosin (HÆE) Staining and Immunohistochemistry Analysis. Testis samples of 7 days' and 2 months' old male pigs were fixed, dehydrated, and embedded in paraffin. The paraffin-embedded tissues were then sectioned at $5 \mu \mathrm{m}$ using standard procedures and adhered to precoated glass slides. Afterwards, H\&E staining of the paraffinembedded sections was conducted to observe the histology [26].

For immunohistochemistry, PDGFR $\alpha$ expression in the interstitial cells of 7-day-old porcine testes and the type of these protein-positive cells was determined. In detail, the paraffin sections were deparaffinized, rehydrated, and rinsed in PBS. Then antigen retrieval involved boiling of the samples in a solution of $0.01 \mathrm{M}$ Tris-ethylenediamine tetraacetic acid (Tris-EDTA; $\mathrm{pH}=9.0$ ) for $10 \mathrm{~min}$. The sections were incubated with $10 \%$ donkey serum for $2 \mathrm{~h}$ at $37^{\circ} \mathrm{C}$, followed by incubation with primary antibodies (anti-PDGFR $\alpha, 1: 200$, Abcam, Cambridge, UK) overnight at $4^{\circ} \mathrm{C}$ and subsequent incubation with secondary biotinylated antibodies (ZSGB$\mathrm{BIO}$, China) for $1 \mathrm{~h}$ at $37^{\circ} \mathrm{C}[27,28]$. Afterwards, 3,3'diaminobenzidine (DAB, ComWin Biotech, China) was used as a chromogen to detect protein expression.

The characteristics of the isolated cells were detected by immunofluorescence staining. First, cells were fixed with $4 \%$ paraformaldehyde and permeabilized with $0.05 \%$ Triton X-100 for $15 \mathrm{~min}$. The cells were then incubated with primary antibodies at $4^{\circ} \mathrm{C}$ overnight, and then for $2 \mathrm{~h}$ with appropriate Alexa Fluor 594-conjugated secondary antibodies (1:400, Invitrogen, USA) at $37^{\circ} \mathrm{C}$. Finally, the cells were labeled with 4,6-diamidino-2-phenylindole (DAPI, 1:1000; Beyotime, China). The primary antibodies used were rabbit anti-PDGFR $\alpha$ (1:200, Abcam) and mouse anti-CYP17A1 ( $1: 100$, Santa Cruz, USA).

All images of all the staining were captured using a Nikon Eclipse 80i fluorescence microscope camera (Tokyo, Japan).

2.7. $q R T-P C R$ Analysis. Total RNA were extracted from cells and porcine testes tissues using RNAiso Plus reagent (TaKaRa, Dalian, China) according to the recommended protocol. The cDNA was then synthesized for reverse transcription PCR (RT-PCR) using the PrimeSript ${ }^{\mathrm{TM}}$ RT reagent Kit (TaKaRa). Specific primers (Table 1 ) were used to characterize the isolated cells. The qRT-PCR reaction system was $20 \mu \mathrm{L}$ in 
TABLE 1: Primer sequences for genes designed and used in this study.

\begin{tabular}{|c|c|c|c|}
\hline Primers & Primer sequences $\left(5^{\prime}-3^{\prime}\right)$ & Length of production/bp & Notes \\
\hline \multirow{2}{*}{ LIFR } & F: TAGCACGTGAATTGCGGACT & \multirow{2}{*}{117} & \multirow{2}{*}{ RT-PCR \& qRT-PCR } \\
\hline & R: CAGTGCAACAACGAATGCGA & & \\
\hline \multirow{2}{*}{ Nestin } & F: GGAGAAACAGGGCCTACAGAG & \multirow{2}{*}{112} & \multirow{2}{*}{ RT-PCR } \\
\hline & R: TAGGAGGGTCCTGTATGTGGC & & \\
\hline \multirow{2}{*}{ GATA-4 } & F: AATCGAAGACGTCAGCAGGT & \multirow{2}{*}{123} & \multirow{2}{*}{ RT-PCR } \\
\hline & R: GCTCTGTCTTGATGGGACGC & & \\
\hline \multirow{2}{*}{ Oct 4} & F: GTGTTCAGCCAAACGACCATC & \multirow{2}{*}{143} & \multirow{2}{*}{ RT-PCR } \\
\hline & R: GTCTCTGCCTTGCATATCTCC & & \\
\hline \multirow{2}{*}{$\operatorname{PDGFR} \alpha$} & F: GTGGAGAATCTGCTGCCTGG & \multirow{2}{*}{133} & \multirow{2}{*}{ RT-PCR \& qRT-PCR } \\
\hline & R: TGTAGGTGACGCCGATGTAG & & \\
\hline \multirow{2}{*}{ PLZF } & F: GCGGAAGACCTGGATGACCT & \multirow{2}{*}{105} & \multirow{2}{*}{ RT-PCR } \\
\hline & R: GTCGTCTGAGGCTTGGATGGT & & \\
\hline \multirow{2}{*}{ SOX9 } & F: GCAAACTCTGGAGACTGCTGAATG & \multirow{2}{*}{137} & \multirow{2}{*}{ RT-PCR } \\
\hline & R: GCCGTTCTTCACCGACTTTCTC & & \\
\hline \multirow{2}{*}{ CYP17A1 } & F: ATTGACTCCAGCATTGGCGA & \multirow{2}{*}{179} & \multirow{2}{*}{ RT-PCR \& qRT-PCR } \\
\hline & R: CCGAAGGGCAAGTAGCTCAA & & \\
\hline \multirow{2}{*}{$\beta$-actin } & F: CTCCATCATGAAGTGCGACGT & \multirow{2}{*}{114} & \multirow{2}{*}{ RT-PCR \& qRT-PCR } \\
\hline & R: GTGATCTCCTTCTGCATCCTGTC & & \\
\hline
\end{tabular}

volume: $10 \mu \mathrm{L}$ SYBR ${ }^{\circledR}$ Premix Ex Taq II (2x) (TaKaRa), $0.8 \mu \mathrm{L}$ cDNA, $0.5 \mu \mathrm{L}$ PCR Forward Primer $(10 \mu \mathrm{mol} / \mathrm{L}), 0.5 \mu \mathrm{L}$ PCR Reverse Primer $(10 \mu \mathrm{mol} / \mathrm{L})$, and added sterile water to total volume of $20 \mu \mathrm{L}$. PCR reaction conditions were as follows: denaturation at $95^{\circ} \mathrm{C}$ for $3 \mathrm{~min}$, followed by 40 cycles of $\left(95^{\circ} \mathrm{C}\right.$ for $15 \mathrm{~s}, 60^{\circ} \mathrm{C}$ for $30 \mathrm{~s}$, and $72^{\circ} \mathrm{C}$ for $30 \mathrm{~s}$ ).

2.8. Oil Red O Staining. For the visualization of the lipid droplets, LC was fixed in 4\% formaldehyde (freshly prepared from paraformaldehyde) for $15 \mathrm{~min}$, stained in Oil red $\mathrm{O}$ staining solution $(0.3 \%$ Oil Red $\mathrm{O}$ solution) for $10 \mathrm{~min}$, and then washed with PBS 2 3 times. The cells were then captured using a Nikon Eclipse 80i fluorescence microscope camera.

2.9. Statistical Analyses. The mRNA expressions detected by qRT-PCR were calculated using the $2^{-\Delta \Delta C T}$ method and normalized by the expression of $\beta$-actin [29]. The mRNA expression variation between different samples was calculated using SPSS (version 18.0) (SPSS, Inc., Chicago, IL, USA). Statistical differences of genes in different groups were determined by ANOVA, and the data were presented as mean \pm standard deviation of duplicates.

\section{Results}

3.1. SLCs Were Present in the Neonatal Porcine Testes. A number of spindle-shaped cells were found in the testicular interstitium in the postnatal 7 days' and 2 months' old porcine testes by H\&E staining (Figure 1(a)). Furthermore, immunochemical analyses showed that PDGFR $\alpha$ was mainly expressed in the testicular interstitium in postnatal 7-dayold pigs, while the expression of PDGFR $\alpha$ was low in the 2-month-old porcine testicular interstitium (Figure 1(b)).
Moreover, the expression of Nestin in the 7-day-old porcine testes was significantly higher than that in the 2-month-old testes $(P<0.5)$ (Figure $1(\mathrm{c}))$. Based on these results, we chose to collect SLCs from 7-day-old pigs rather than 2-month-old pigs.

3.2. The Isolated LCs from Porcine Testicular Interstitium Expressed Markers of SLCs. The primary LCs were obtained by digestion method (Figure 2(a)). RT-PCR and immunofluorescent analysis were then used to characterize these cells. As shown in Figure 2(b), RT-PCR results showed that the isolated LCs expressed SLCs or pluripotency stem cell markers (Nestin, PDGFR $\alpha$, GATA-4, Oct4, and LIFR) (Figure 2(b)). Moreover, markers of Sertoli cells (SOX9) and spermatogonial stem cells (PLZF) were not detected (Figure 2(b)), indicating no contamination with these cells in these LCs. The results demonstrated that this digestion method was useful in removing the seminiferous tubules. Moreover, qRT-PCR results showed that the expressions of LIFR and PDGFR $\alpha$ in the LCs were significantly higher than that in the porcine testes $(P<0.5)$ (Figures $2(\mathrm{c})$ and $2(\mathrm{~d})$ ), indicating that this method was able to enrich SLCs from porcine testes. In summary, the primary isolated LCs, expressing SLCs markers (Nestin, PDGFR $\alpha$, GATA-4, Oct4, and LIFR), were putative SLCs.

EDS was used to specifically eliminate differentiated LCs in rat and mouse testes $[4,8]$. According to results of the EDS treatment, the percentage of porcine differentiated LCs was approximately $23 \%$ in the primary isolated LCs, and the purity of primary isolated porcine SLCs was over $77 \%$ (Figures S1, S2, in Supplementary Material available online at https://doi.org/10.1155/2017/2740272). In addition, qRT-PCR results of Nestin, PDGFR $\alpha, C Y P 17 A 1$ expressions, and the 

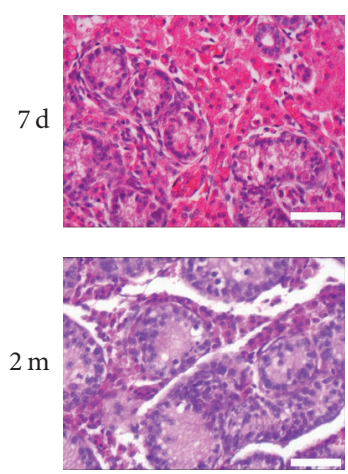

(a)
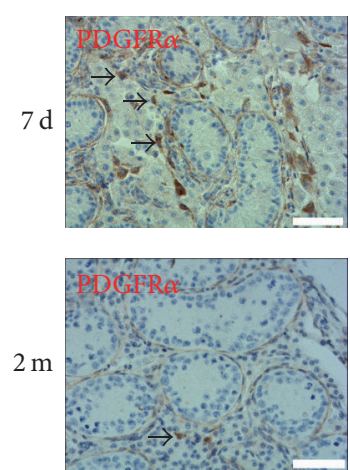

(b)
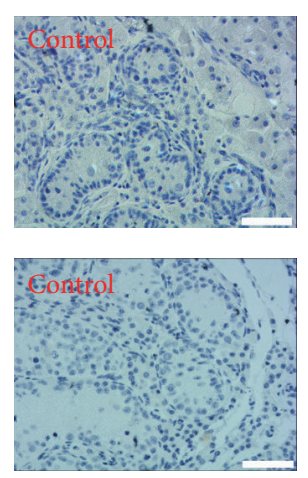

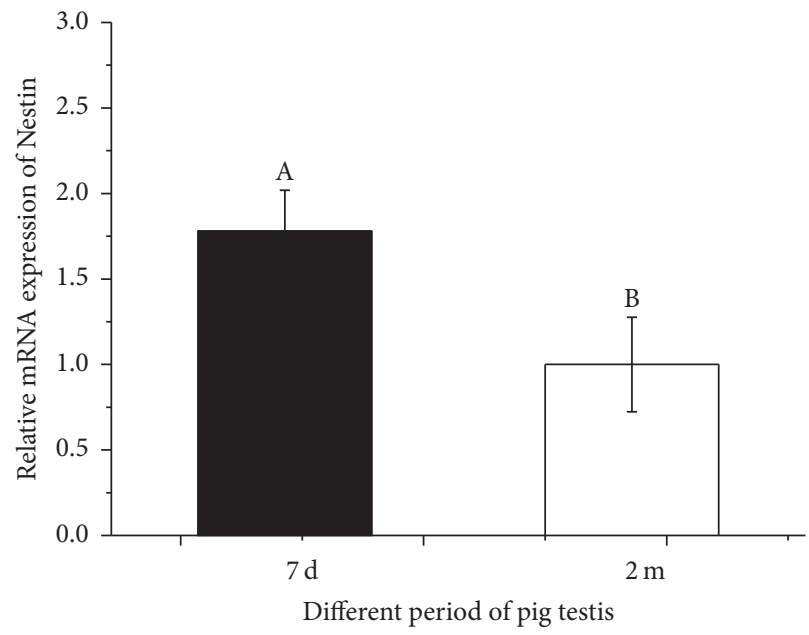

(c)

Figure 1: Identification of pig stem Leydig cells (SLCs) in situ. (a) H\&E staining of 7 days' and 2 months' old porcine testes (bar $=50 \mu \mathrm{m}$ ). (b) Immunohistochemical analysis of PDGFR $\alpha$ of 7 days' and 2 months' old porcine testes ( $\mathrm{bar}=50 \mu \mathrm{m})$; the black arrowheads indicated the PDGFR $\alpha$-positive cells in testicular interstitium. (c) mRNA expression of Nestin in 7 days' and 2 months' old pig testes. Different letters (A, B) indicate significant difference $(P<0.05)$.

immunofluorescent analysis of CYP17A1 further confirmed that EDS could specifically eliminate differentiated LCs in the pig (Figures S3, S4), which was consistent with the results of cell survival rates after EDS treatment (Figure S2).

\subsection{These Isolated SLCs Exhibited High Clonogenic Potential.} Even though the primary SLCs were isolated, their culture system was yet to be determined. In the current study, pTF was used as the main component in the medium. Seven days later, a number of clones were formed, which grew larger following 2 weeks of culture (Figure 3(a)). Immunofluorescent analysis showed that the clones were PDGFR $\alpha$ positive (Figure 3(b)). The expressions of both Nestin and LIFR were higher in porcine SLCs cultured with pTF medium compared to in SLCs without culture $(P<0.5)$ (Figure 5), indicating that pTF was able to sustain the stem cell potential of SLCs.

\subsection{Isolated SLCs Showed the Capacity of Spontaneous Differ-} entiation into LCs When Cultured In Vitro. The isolated cells cultured with a basic medium did not form clones after 2 weeks (Figure 4(a)) and expressed CYP17A1, a marker of pig differentiated LCs (Figure 4(b)). Moreover, the expressions of both Nestin and LIFR were significantly lower in porcine SLCs cultured with the basic medium for 2 weeks compared to in SLCs without culture $(P<0.5)$ (Figure 5). The expression of CYP17A1 was significantly higher in porcine SLCs cultured with the basic medium for 2 weeks than that of SLCs without culture $(P<0.5)$ (Figure 5). Oil Red $\mathrm{O}$ staining showed that the cultured cells secreted lipid droplets, which was also a marker of differentiated LCs (Figure 6). These results demonstrated that the primary isolated SLCs were able to differentiate into LC lineages when cultured with the basic medium, indicating that the putative SLCs had capacity to spontaneously differentiate into LCs.

\section{Discussion}

Several cell types were essential for spermatogenesis in the testis: germ cells, Sertoli cells, peritubular myoid cells, and ALCs [30]. ALCs were the major source of testosterone secretion in mammals; however, they were incapable of proliferation. Testosterone could diffuse into Sertoli cells so that it indirectly regulated spermatogenesis. When the 


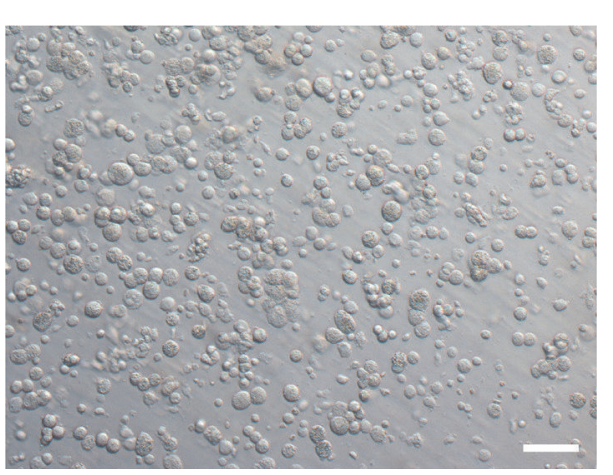

(a)

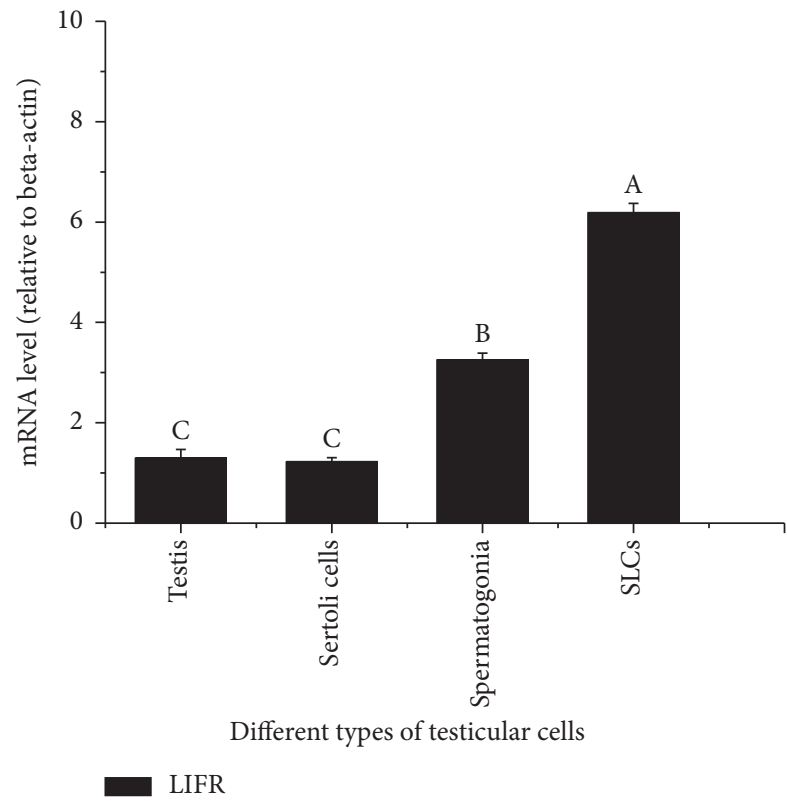

(c)

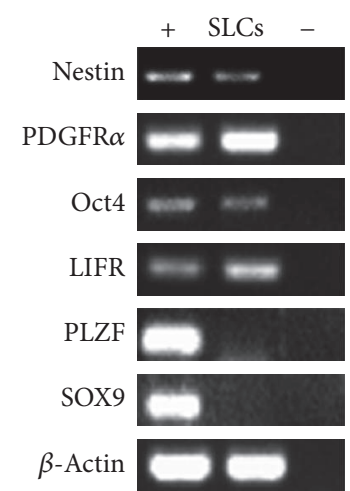

(b)

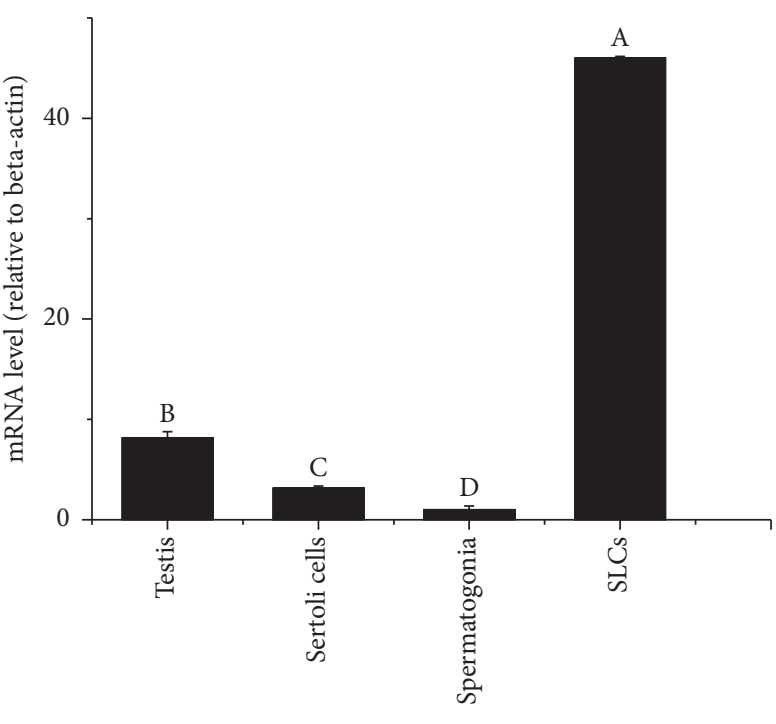

PDGFR $\alpha$

(d)

FIGURE 2: Identification of porcine SLCs. (a) The primary SLCs isolated from 7-day-old porcine testes with the help of collagenase type IV (bar $=50 \mu \mathrm{m}$ ). (b) RT-PCR results of genes involved in stem cells potential and spermatogenesis; SLCs, the pig primary isolated SLCs; +, positive control (7-day-old pig testes); -, negative control (sterile water). (c and d) Expressions of LIFR and PDGFR $\alpha$ in pig testes, pig Sertoli cells, pig Spermatogonia stem cells and pig primary isolated SLCs as fold change relative to beta-actin; Spermatogonia, Spermatogonia stem cells. Different letters $(\mathrm{A}, \mathrm{B})$ indicate significant difference $(P<0.05)$.

processes of synthesizing testosterone were disturbed, postmeiotic spermatids were significantly reduced or absent [30]. SLCs were therefore ideal for rescuing infertility caused by LCs dysfunction. In addition, it had been demonstrated that SLCs were able to differentiate into ALCs in vivo by transplanting the alginate-encapsulated interstitial tissue into rat extra-testis tissue [31]. Therefore, mammalian SLCs held great promise for research and clinical use in male infertility.

Recently, SLCs had been successfully isolated from rats, mice, and humans, but not from pigs. Previous studies had shown that several proteins were detected in putative SLCs in the rat testicular interstitium, such as Nestin, LIFR, $\operatorname{PDGFR} \alpha, \mathrm{CD} 90$, and CD51 [11, 32]. However, a majority of these were also expressed in other testicular cells, and they made useful markers of SLCs, as they were expressed in a time and/or stage-specific manner. For example, Ge and his colleagues demonstrated that the PDGFR $\alpha$-positive and $3 \beta$-HSD-negative cells in postnatal 7-day-old rats were putative SLCs [4]. They then concluded that PDGFR $\alpha$ was a marker of rat SLCs in the neonatal stage. In this study, SLCs were identified and PDGFR $\alpha$ was shown to be expressed in the SLCs using $\mathrm{H} \& \mathrm{E}$ staining and immunochemistry. Moreover, results from immunochemistry and qRT-PCR analysis showed that the expressions of both PDGFR $\alpha$ and Nestin were significantly higher in postnatal 7 days' than 2 months' old pig $(P<0.5)$. These results predicted that PDGFR $\alpha$ could also be used as a marker of neonatal porcine SLCs and the 7-day-old sampling point was more suitable for isolating SLCs than the 2 months old in pigs.

However, no studies had reported the isolation of porcine SLCs. In the rat, Percoll purification and immunoselection technologies were used to obtain SLCs by Ge et al. (2006) [4], 

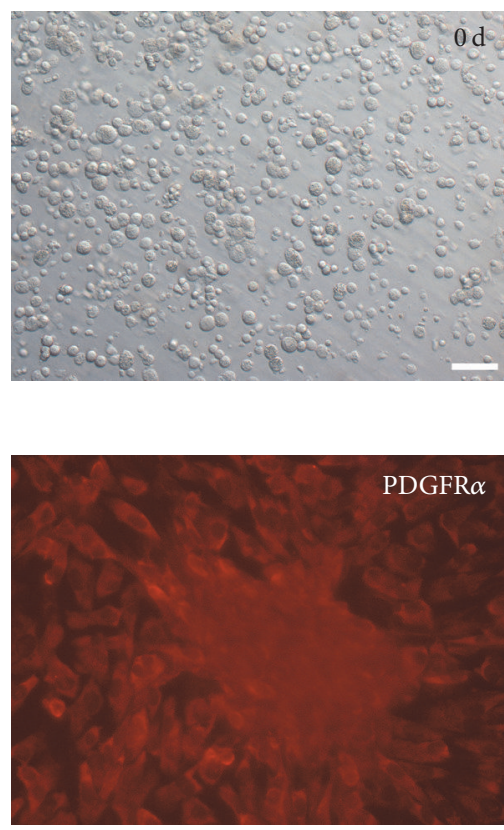

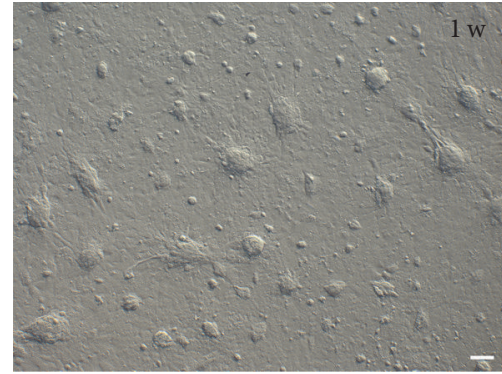

(a)

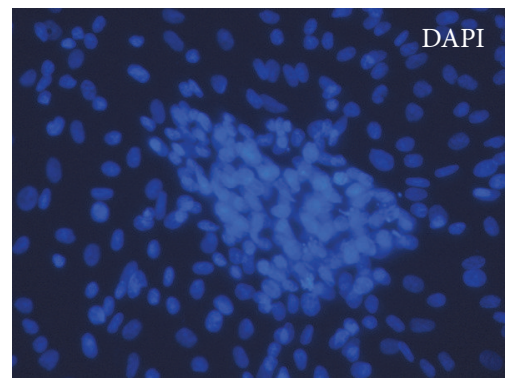

(b)
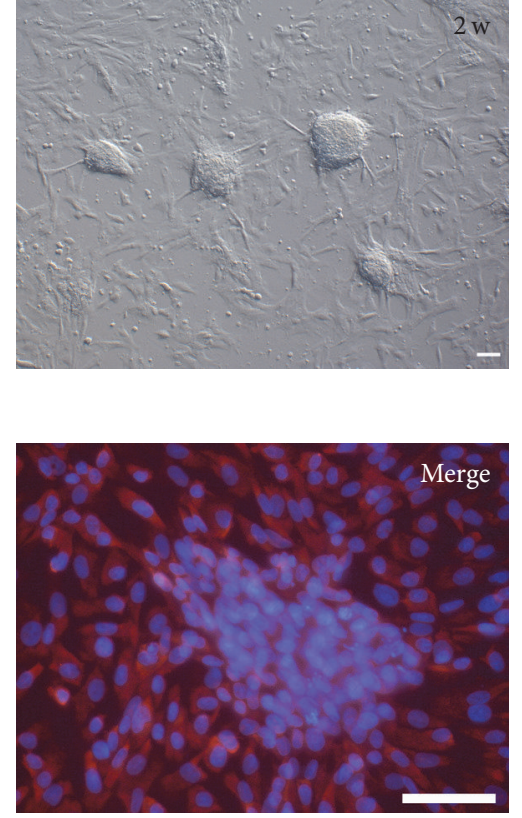

FIGURE 3: Morphology development and PDGFR $\alpha$ immunofluorescence analysis of porcine SLCs cultured in pTF medium $(\mathrm{bar}=50 \mu \mathrm{m})$. (a) Morphology development of porcine SLCs cultured $0 \mathrm{~d}, 1 \mathrm{w}$, and $2 \mathrm{w}$ in pTF medium. (b) PDGFR $\alpha$ immunofluorescence of porcine SLCs cultured in PTF medium for $2 \mathrm{w}$.
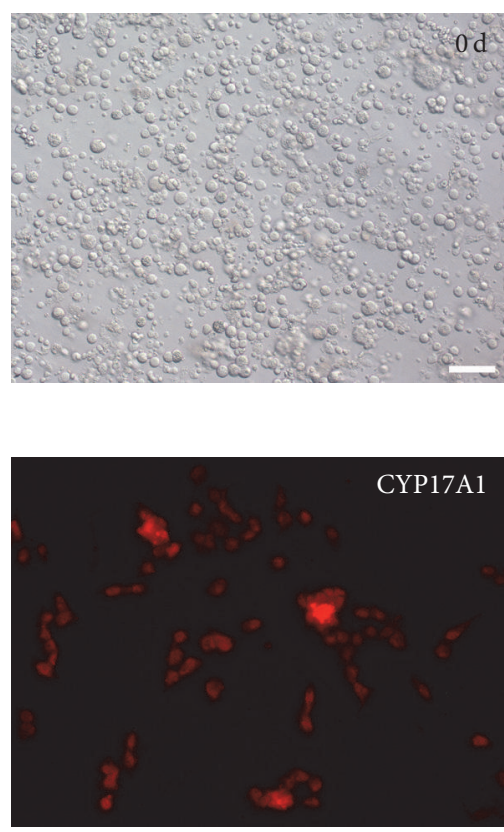
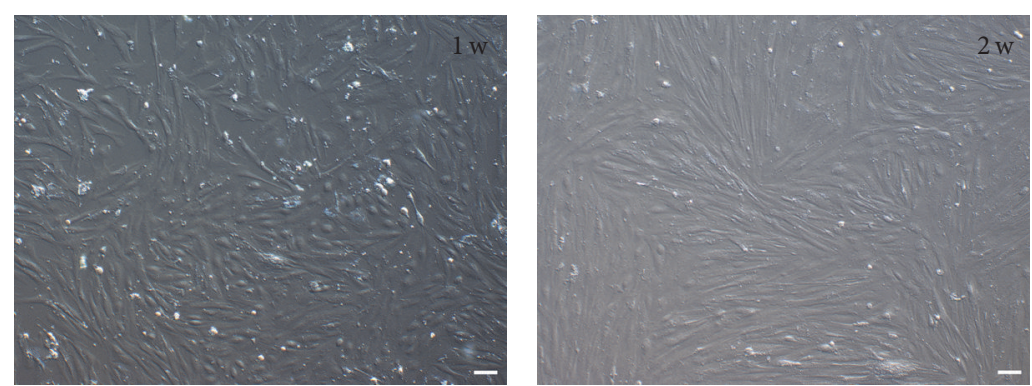

(a)
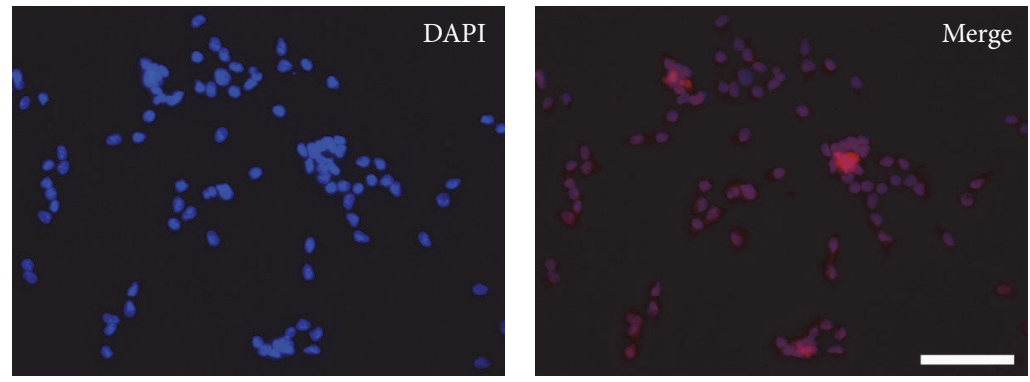

(b)

FIGURE 4: Morphology development and CYP17A1 immunofluorescence of porcine SLCs cultured in basic medium. (a) Morphology development of porcine SLCs cultured $0 \mathrm{~d}, 1 \mathrm{w}$, and $2 \mathrm{w}$ in basic medium. (b) CYP17A1 immunofluorescence of porcine SLCs cultured in basic medium for $2 \mathrm{w}$.

and several studies had used transgenic mice to obtain mouse SLCs $[8,9]$. In the current study, collagenase and hyaluronidase digestion was used to isolate pig testicular interstitial cells from pig testes. Moreover, hyaluronidase could isolate individual cells from the outer surface of seminiferous tubules. Thus, the method used in the current study was simpler and faster than the methods used in mice and rats.

Like other stem cells, the proliferation and differentiation of SLCs were also regulated by the microenvironment, which 


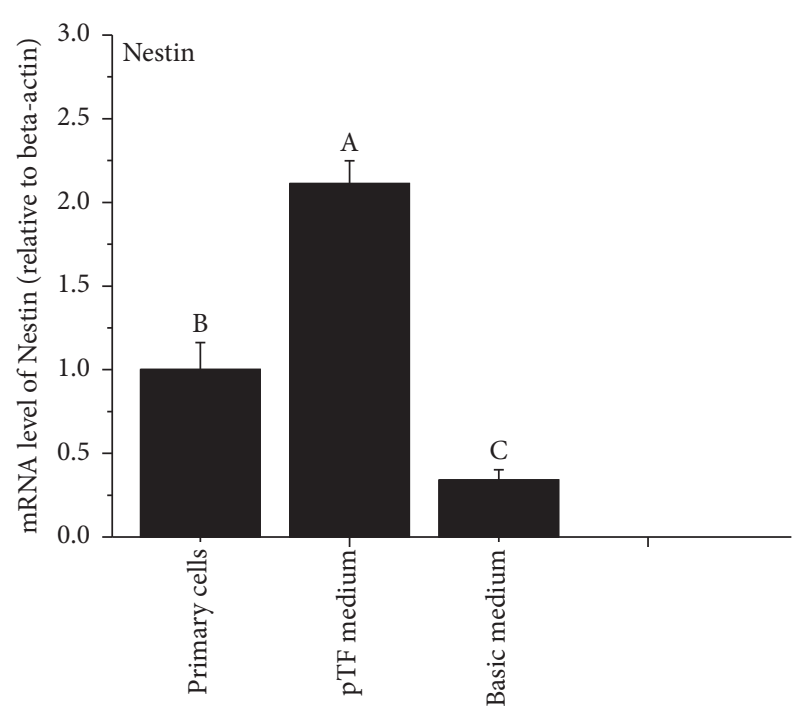

(a)

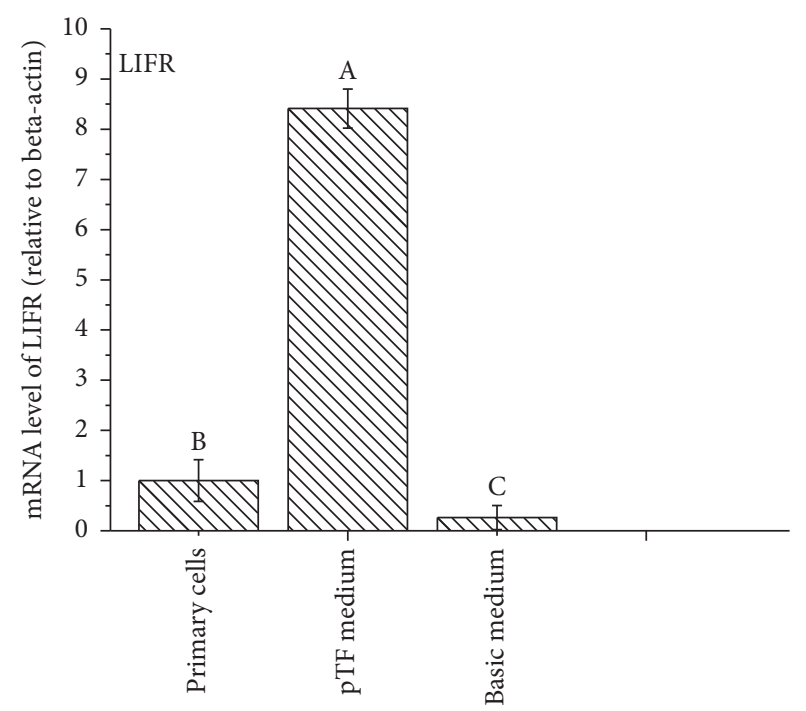

(b)

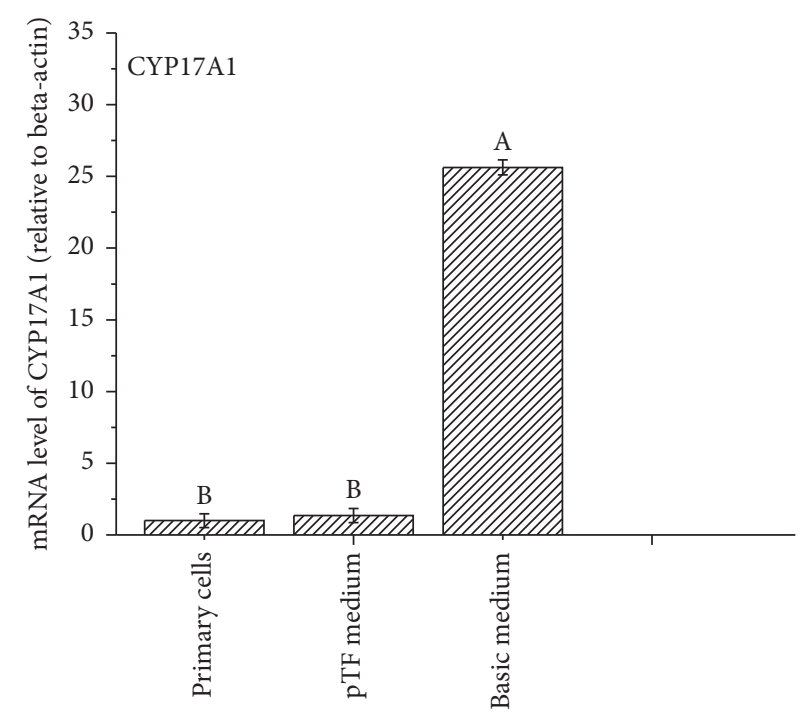

(c)

Figure 5: Expressions of Nestin, LIFR, and CYP17A1 of porcine SLCs cultured in different media for 2 w. Note: primary cells, the primary isolated porcine SLCs. Different letters $(A, B, C)$ indicate significant difference $(P<0.05)$.

provided vital cell factors and proteins. In the testes, some types of cells, such as Sertoli cells and peritubular myoid cells, secreted factors into the testicular fluid to regulate the activities of SLCs [33-35]. Since the culture system of porcine SLCs had not been developed, all factors from whole testes were extracted as pTF. At first, we conjectured that the pTF could maintain the stem cell potential of porcine SLCs when added to the culture medium. The results of this work showed that the pTF could indeed support the stem cell potential of SLCs for 2 weeks in vitro. The pTF was able to maintain the self-renewal properties of SLCs, as the origin of pTF was consistent with the putative SLCs. Moreover, the pTF contained abundant hormones, growth factors, cytokines, and a large amount of proteins, which could provide the necessary material basis for SLCs proliferation $[22,36]$. The immunofluorescent analysis of PDGFR $\alpha$ also demonstrated that the cells that had been cultured for 2 weeks were putative SLCs. Taken together, the results indicated that the pTF might be contributing to maintaining self-renewal properties of the putative SLCs. Therefore, our future research will be directed towards revealing the vital components for maintaining SLCs self-renewal in pTF.

There were two areas of innovation of the present study. First, it provided a simpler and faster method for obtaining the porcine SLCs, which might provide a reservoir for LCslineage differentiation. Second, it developed a new shortterm culture system for porcine SLCs. In addition, as an ideal human model, some human drugs toxicity investigations of 


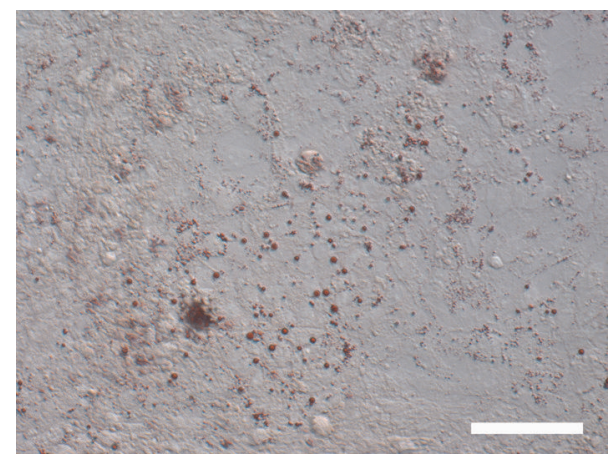

FIGURE 6: Oil Red O staining of pig LCs after cultured $7 \mathrm{~d}$ in the basic medium (bar $=50 \mu \mathrm{m})$.

sterile diseases could be assessed in the pig firstly, before human trials, which could reduce the expense of investigations into new drugs.

\section{Conclusions}

To summarize, we isolated porcine SLCs and identified some of their basic characteristics. Moreover, pTF could maintain the features of porcine SLCs when added to culture system. This work might help us to understand the regulatory mechanisms of proliferation and differentiation of SLCs and holds promise for further studies pertaining to porcine SLCs.

\section{Disclosure}

The current address of Chuanying Pan is College of Animal Science and Technology, Northwest A\&F University, No. 22 Xinong Road, Yangling, Shaanxi 712100, China.

\section{Competing Interests}

The authors declare that there are no competing interests regarding the publication of this paper.

\section{Acknowledgments}

This work was supported by China Postdoctoral Science Foundation funded project (no. 2014M560809), the Fundamental Research Funds for the Central Universities (NWSUAF, no. 2452015145), and the National Basic Research Program of China (973 program; no. 2014CB943100). Special thanks are due to Professor Yuan-Qiang Zhang (The Fourth Military Medical University, China) for his generous donation of EDS.

\section{References}

[1] A. S. Midzak, H. Chen, V. Papadopoulos, and B. R. Zirkin, "Leydig cell aging and the mechanisms of reduced testosterone synthesis," Molecular and Cellular Endocrinology, vol. 299, no. 1, pp. 23-31, 2009.
[2] Y. Yang, Z. Su, W. Xu et al., "Directed mouse embryonic stem cells into leydig-like cells rescue testosterone-deficient male rats in vivo," Stem Cells and Development, vol. 24, no. 4, pp. 459-470, 2015.

[3] H. Chen, R.-S. Ge, and B. R. Zirkin, "Leydig cells: from stem cells to aging," Molecular and Cellular Endocrinology, vol. 306, no. 1-2, pp. 9-16, 2009.

[4] R.-S. Ge, Q. Dong, C. M. Sottas, V. Papadopoulos, B. R. Zirkin, and M. P. Hardy, "In search of rat stem Leydig cells: identification, isolation, and lineage-specific development," Proceedings of the National Academy of Sciences of the United States of America, vol. 103, no. 8, pp. 2719-2724, 2006.

[5] M. S. Davidoff, R. Middendorff, G. Enikolopov, D. Riethmacher, A. F. Holstein, and D. Müller, "Progenitor cells of the testosterone-producing Leydig cells revealed," Journal of Cell Biology, vol. 167, no. 5, pp. 935-944, 2004.

[6] M. S. Davidoff, R. Middendorff, D. Müller, and A. F. Holstein, "The neuroendocrine Leydig cells and their stem cell progenitors, the pericytes," Advances in Anatomy, Embryology, and Cell Biology, vol. 205, pp. 1-107, 2009.

[7] L. Landreh, K. Spinnler, K. Schubert et al., "Human testicular peritubular cells host putative stem leydig cells with steroidogenic capacity," The Journal of Clinical Endocrinology and Metabolism, vol. 99, no. 7, pp. E1227-E1235, 2014.

[8] M. H. Jiang, B. Cai, Y. Tuo et al., "Characterization of Nestinpositive stem Leydig cells as a potential source for the treatment of testicular Leydig cell dysfunction," Cell Research, vol. 24, no. 12, pp. 1466-1485, 2014.

[9] K. C. Lo, Z. Lei, C. Venkateswara Rao, J. Beck, and D. J. Lamb, "De novo testosterone production in luteinizing hormone receptor knockout mice after transplantation of Leydig stem cells," Endocrinology, vol. 145, no. 9, pp. 4011-4015, 2004.

[10] E. Stanley, C.-Y. Lin, S. Jin et al., "Identification, proliferation, and differentiation of adult leydig stem cells," Endocrinology, vol. 153, no. 10, pp. 5002-5010, 2012.

[11] X. Li, Z. Wang, Z. Jiang et al., "Regulation of seminiferous tubule-associated stem Leydig cells in adult rat testes," Proceedings of the National Academy of Sciences of the United States of America, vol. 113, no. 10, pp. 2666-2671, 2016.

[12] L. Luo, H. Chen, and B. R. Zirkin, “Temporal relationships among testosterone production, steroidogenic acute regulatory protein (StAR), and P450 side-chain cleavage enzyme (P450scc) during Leydig cell aging," Journal of Andrology, vol. 26, no. 1, pp. 25-31, 2005.

[13] A. V. Pechersky, V. F. Semiglazov, G. B. Loran, A. I. Karpischenko, V. I. Pechersky, and V. I. Mazurov, "The influence of partial androgen deficiency of aging men (PADAM) on the impulse regime of incretion of several hormones and mitotic activity," Tsitologiya, vol. 48, no. 10, pp. 862-866, 2006.

[14] M. Amore, F. Scarlatti, A. L. Quarta, and P. Tagariello, "Partial androgen deficiency, depression and testosterone treatment in aging men," Aging Clinical and Experimental Research, vol. 21, no. 1, pp. 1-8, 2009.

[15] A. Pechersky, "Features of diagnostics and treatment of partial androgen deficiency of aging men," Central European Journal of Urology, vol. 67, no. 4, pp. 397-404, 2014.

[16] Y. Nian, M. Ding, S. Hu et al., "Testosterone replacement therapy improves health-related quality of life for patients with late-onset hypogonadism: a meta-analysis of randomized controlled trials," Andrologia, 2016. 
[17] Y. Zhang, R. Ge, and M. P. Hardy, "Androgen-forming stem Leydig cells: identification, function and therapeutic potential," Disease Markers, vol. 24, no. 4-5, pp. 277-286, 2008.

[18] S. Bergfelder-Drüing, C. Grosse-Brinkhaus, B. Lind et al., "A genome-wide association study in large white and landrace pig populations for number piglets born alive," PLoS ONE, vol. 10, no. 3, Article ID e0117468, 2015.

[19] Z. Jiang and M. F. Rothschild, "Swine genome science comes of age," International Journal of Biological Sciences, vol. 3, no. 3, pp. 129-131, 2007.

[20] J. I. Raeside, H. L. Christie, R. L. Renaud, and P. A. Sinclair, "The boar testis: the most versatile steroid producing organ known," Society of Reproduction and Fertility Supplement, vol. 62, pp. 8597, 2006.

[21] Y. Nakajima, G. Sato, S. Ohno, and S. Nakajin, "Organotin compounds suppress testosterone production in Leydig cells from neonatal pig testes," Journal of Health Science, vol. 49, no. 6, pp. 514-519, 2003.

[22] P. Wang, Y. Zheng, Y. Li et al., "Effects of testicular interstitial fluid on the proliferation of the mouse spermatogonial stem cells in vitro," Zygote, vol. 22, no. 3, pp. 395-403, 2014.

[23] E.-H. Lee, J.-H. Oh, Y.-S. Lee et al., "Gene expression analysis of toxicological pathways in TM3 leydig cell lines treated with Ethane dimethanesulfonate," Journal of Biochemical and Molecular Toxicology, vol. 26, no. 6, pp. 213-223, 2012.

[24] T. Li, J. Hu, G.-H. He et al., "Up-regulation of NDRG2 through nuclear factor-kappa B is required for Leydig cell apoptosis in both human and murine infertile testes," Biochimica et Biophysica Acta-Molecular Basis of Disease, vol. 1822, no. 2, pp. 301-313, 2012.

[25] A. J. Morris, M. F. Taylor, and I. D. Morris, "Leydig cell apoptosis in response to ethane dimethanesulphonate after both in vivo and in vitro treatment," Journal of Andrology, vol. 18, no. 3, pp. 274-280, 1997.

[26] B. Heidari, M. Rahmati-Ahmadabadi, M. M. Akhondi et al., "Isolation, identification, and culture of goat spermatogonial stem cells using c-kit and PGP9.5 markers," Journal of Assisted Reproduction and Genetics, vol. 29, no. 10, pp. 1029-1038, 2012.

[27] J.-P. Qi, Y.-L. Yang, H. Zhu et al., "Expression of the androgen receptor and its correlation with molecular subtypes in $980 \mathrm{Chi-}$ nese breast cancer patients," Breast Cancer: Basic and Clinical Research, vol. 6, pp. 1-8, 2012.

[28] Y. Zheng, Y. He, J. An et al., "THY1 is a surface marker of porcine gonocytes," Reproduction, Fertility and Development, vol. 26, no. 4, pp. 533-539, 2014.

[29] K. J. Livak and T. D. Schmittgen, "Analysis of relative gene expression data using real-time quantitative PCR and the 2(T)(-Delta Delta C) method," Methods, vol. 25, no. 4, pp. 402408, 2001.

[30] L. B. Smith and W. H. Walker, "The regulation of spermatogenesis by androgens," Seminars in Cell and Developmental Biology, vol. 30, pp. 2-13, 2014.

[31] H. Chen, S. Jin, S. Huang et al., "Transplantation of alginateencapsulated seminiferous tubules and interstitial tissue into adult rats: leydig stem cell differentiation in vivo?" Molecular and Cellular Endocrinology, vol. 436, pp. 250-258, 2016.

[32] H. Chen, Y. Wang, R. Ge, and B. R. Zirkin, "Leydig cell stem cells: identification, proliferation and differentiation," Molecular and Cellular Endocrinology, 2016.

[33] R. M. Sharpe, "Monitoring of spermatogenesis in manmeasurement of Sertoli cell- or germ cell-secreted proteins in semen or blood," International Journal of Andrology, vol. 15, no. 3, pp. 201-210, 1992.

[34] K. J. Turner, C. McKinnell, T. T. McLaren et al., "Detection of germ cell-derived proteins in testicular interstitial fluid: potential for monitoring spermatogenesis in vivo," Journal of Andrology, vol. 17, no. 2, pp. 127-136, 1996.

[35] L. R. França, R. A. Hess, J. M. Dufour, M. C. Hofmann, and M. D. Griswold, "The Sertoli cell: one hundred fifty years of beauty and plasticity," Andrology, vol. 4, no. 2, pp. 189-212, 2016.

[36] P. G. Stanton, C. F. Foo, A. Rainczuk et al., "Mapping the testicular interstitial fluid proteome from normal rats," PROTEOMICS, vol. 16, no. 17, pp. 2391-2402, 2016. 

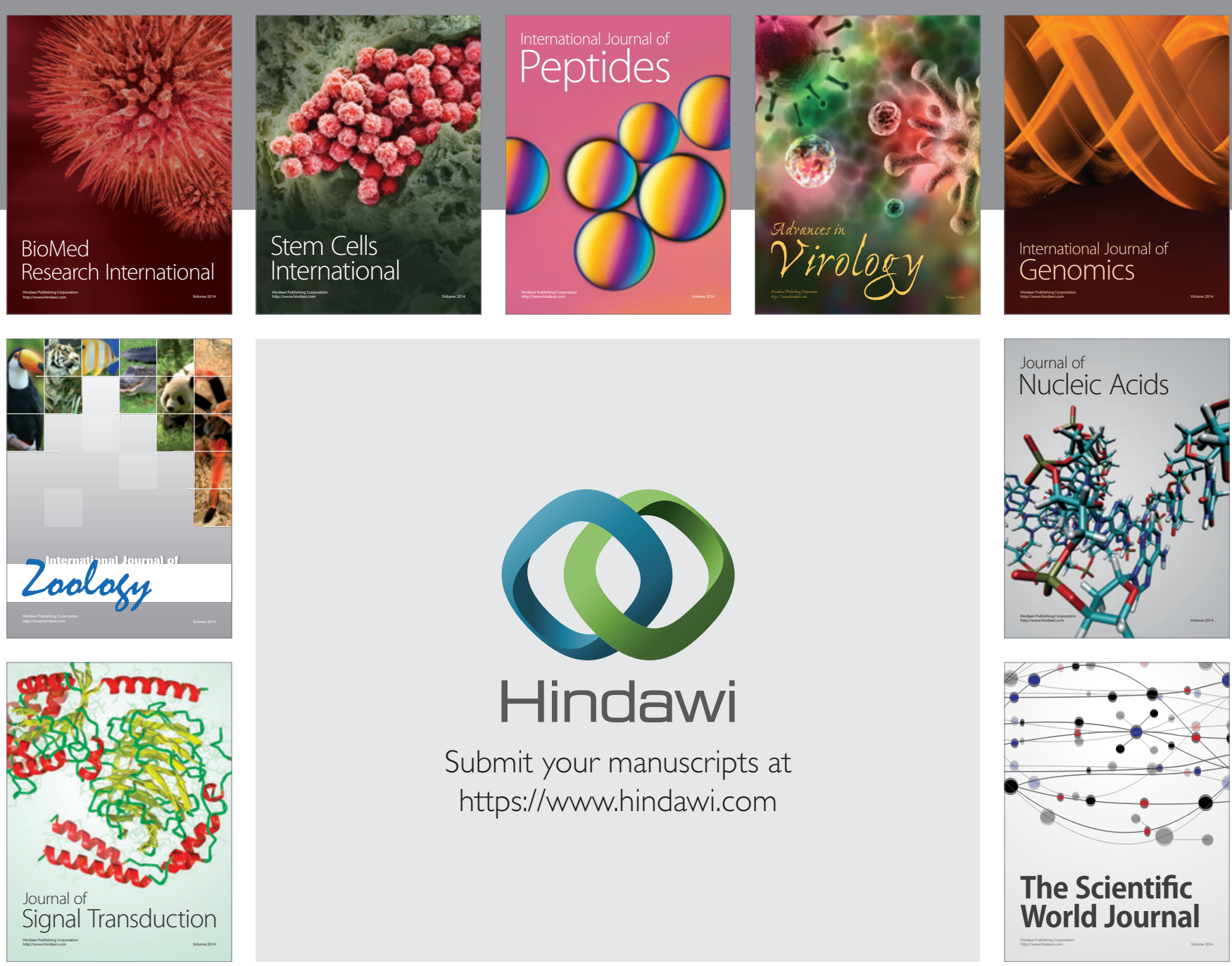

Submit your manuscripts at

https://www.hindawi.com
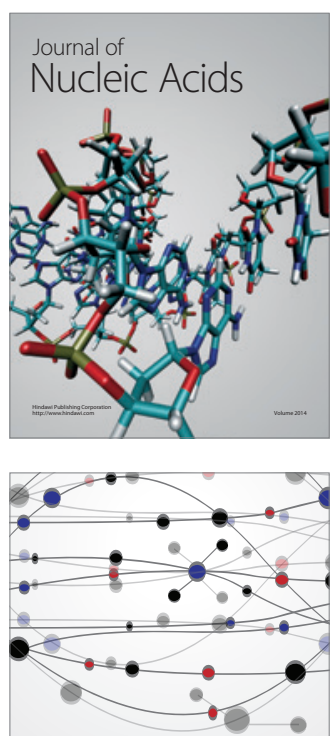

The Scientific World Journal
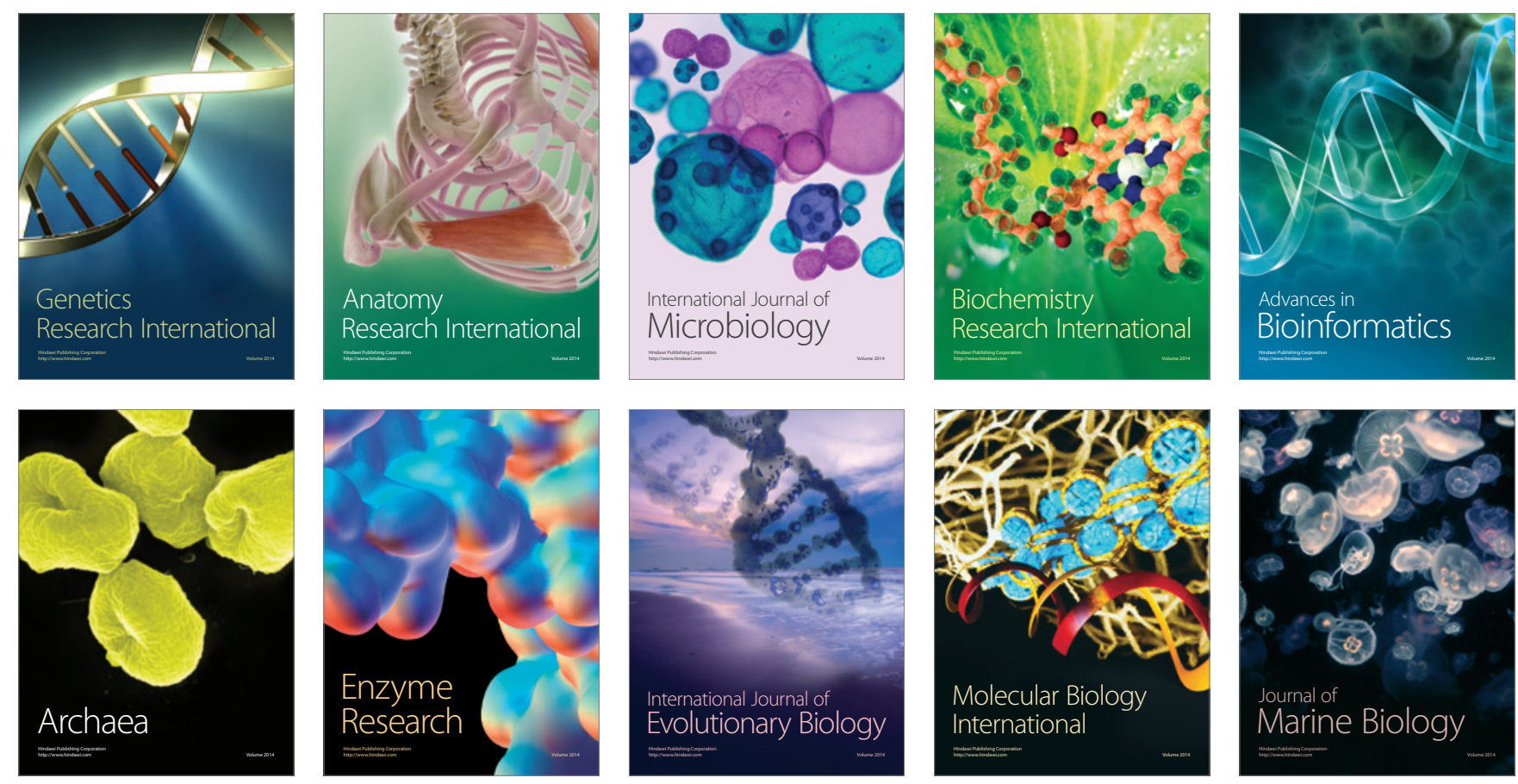